\title{
A 3-D model-based registration approach for the PET, MR and MCG cardiac data fusion
}

\author{
Timo Mäkelä a b c *, Quoc Cuong Pham ${ }^{\text {b }}$, Patrick Clarysse ${ }^{\text {b }}$, Jukka Nenonen ${ }^{\text {a c }}$, \\ Jyrki Lötjönen d, Outi Sipilä e, Helena Hänninen ${ }^{\mathrm{f}}$, Kirsi Lauerma ${ }^{\text {e, }}$ \\ Juhani Knuuti ${ }^{\mathrm{g}}$, Toivo Katila ${ }^{\text {a }}{ }^{\text {and Isabelle E. Magnin }}{ }^{\mathrm{b}}$ \\ ${ }^{a}$ Laboratory of Biomedical Engineering, Helsinki University of Technology, \\ P.O.B. 2200, FIN-02015 HUT, Finland \\ ${ }^{\mathrm{b}}$ CREATIS, INSA, Batiment Blaise Pascal, 69621 Villeurbanne Cedex, France \\ ${ }^{\mathrm{c} B i o M a g}$ Laboratory, Helsinki University Central Hospital, P.O.B. 503, FIN-00029 HUS, Finland \\ ${ }^{\mathrm{d}}$ VTT Information Technology, P.O.Box 1206, FIN-33101 Tampere, Finland \\ ${ }^{\text {e }}$ Department of Radiology, Helsinki University Central Hospital, P.O.B. 340, FIN-00029 HUS, Finland \\ ${ }^{\mathrm{f}}$ Division of Cardiology, Helsinki University Central Hospital, P.O.B. 340, FIN-00029 HUS, Finland \\ gTurku PET Centre, c/o Turku University Central Hospital, Box 52, FIN-20521, Finland
}

In this paper, a new approach is presented for the assessment of a 3-D anatomical and functional model of the heart including structural information from magnetic resonance imaging (MRI) and functional information from positron emission tomography (PET) and magnetocardiography (MCG). The method uses model-based coregistration of MR and PET images and marker-based registration for MRI and MCG. Model-based segmentation of MR anatomical images results in an individualized 3-D biventricular model of the heart including functional parameters from PET and MCG in an easily interpretable 3-D form.

\section{Introduction}

Ischemic diseases and their dramatic consequence, the myocardial infarct, are the leading cause of mortality in industrial countries. From the physio-pathological point of view, ischemia results from a disequilibrium between myocardial perfusion, metabolism and contractile function. Following an ischemic event, the question of myocardial viability arises. Both ischemia diagnosis and viability estimation rely on the joint

\footnotetext{
*This study was partly granted by the Scientific Department of the French Embassy in Finland, the Region Rhône Alpes, through the ADéMO project and The Foundation of Technology in Finland. This work was performed within the framework of the joint incentive action "Beating Heart" of the research groups ISIS, ALP and MSPC of the French National Center for Scientific Research (CNRS).
}

analysis of the perfusion, metabolism, and contractile function, each of which being quantified with specific imaging modalities (Fig. 1). Consequences onto the electric and magnetic activity of the heart have also been observed. Therefore, computer-based methods are required to automatically perform multi-modal cardiac image registration in $3-\mathrm{D}$. This is the purpose of this work.

Anatomical (structural) information of the heart is usually provided by Magnetic Resonance Imaging (MRI) and ultrasound (US). Metabolism can be analyzed with Positron Emission Tomography (PET), perfusion with thallium Single Photon Emission Computed Tomography (SPECT), MRI, or PET, and contractile function by using MRI and US. Fluorodeoxyglucose (FDG) PET 


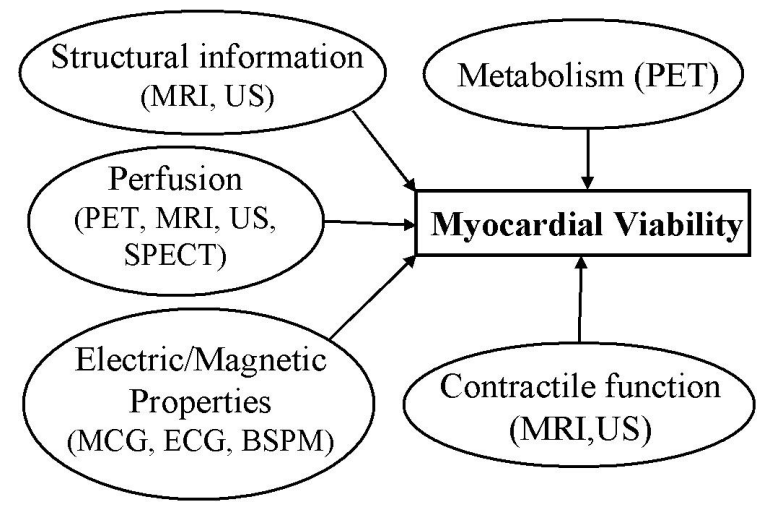

Figure 1. Variety of imaging modalities required for the cardiac viability assessment.

imaging is considered as the gold standard to determine viable areas of the heart (Hartiala and Knuuti, 1995). The electrical activity of the heart creates both an electric and a magnetic field and can be measured by using electrocardiography (ECG) and magnetocardiography (MCG), respectively (Siltanen, 1988). In MCG and ECG inverse problems, a current distribution inside of the heart is estimated from noninvasively measured signals by solving a regularized inverse problem (Hämäläinen and Nenonen, 1999; MacLeod and Brooks, 1998). Thus, multichannel MCG and ECG studies can be applied in locating abnormal electrical activity in the heart (Nenonen et al., 2001). The MCG signals are generated by the same bioelectric currents as the ECG, but the MCG may show ischemia-induced deviations from the normal direction of depolarization and repolarization in a different way than ECG (Hänninen et al., 2001). In the literature, electrical activity and metabolism have usually been studied separately, principally because the acquisition system are quite different and involve different specialists. From the physiopathological point of view, it is clear that all the activities are related one to each other. Therefore, our objective here was to allow for the study of the correlation between the electrical (MCG data) and metabolic (PET imaging) activities which may reveal new aspects of hearts complex pathological processes. To our knowledge, this kind of comparison has not been done before. The presented 3-D method gives good possibilities especially for visual comparison of data from different imaging modalities. Whereas PET imaging is used to study the cardiac metabolism, the multichannel ECG and MCG mappings allow a comprehensive study of the electromagnetic fields of the heart. These mapping techniques give unique additional information on the electromagnetic manifestations of myocardial ischemia and viability. At present, the nuclear imaging modalities are mainly used in elective manner, applied only rarely in clinical decisions concerning cardiac care unit patients, whereas ECG monitoring is widely used in emergency care units. In the future, technical development will hopefully allow the MCG mapping function as clinical tool without need for shielding. Multichannel ECG and MCG studies, used with standard torso models (to obtain individual torso model one needs usually MR images), could then give estimation of ischemia (and viability). The $3-\mathrm{D}$ model based approaches could be first used with functional data from ECG and/or MCG, and then be extended during the clinical studies with other modalities, such as MR and nuclear medicine studies. In this paper, we will use MRI for obtaining the heart's anatomy. Myocardial functional data are issued from FDG-PET for the metabolism and MCG data for the heart's electrical activity.

In viability studies, mental registration of the information from different imaging modalities is routinely performed by clinicians. Automatic registration, based on computer programs, is however expected to offer better accuracy, repeatability, and to save time. Registration of cardiac images is a more complex problem than brain image registration because of the mixed motions of the heart and the thorax structures. Moreover, as compared to the registration of brain images, the heart exhibits much fewer accurate anatomical landmarks and the images are usually acquired with lower resolution. A review of cardiac image registration approaches can be found in (Mäkelä et al., 2002a). These methods are usually based on manual intervention (Behloul et al., 
2001; Waiter et al., 2000), automatic registration of thorax surfaces (Pallotta et al., 1995; Gilardi et al., 1998; Yu et al., 1995; Tai et al., 1997; Cai et al., 1999; Mäkelä et al., 2001a) or heart surfaces (Faber et al., 1991; Sinha et al., 1995; Andersson et al., 1995; Declerck et al., 1997; Thirion, 1995, 2001; Nekolla et al., 2000). Another category of methods relies on the matching of image intensities (Hoh et al., 1993; Bettinardi et al., 1993; Slomka et al., 1995; Eberl et al., 1996; Dey et al., 1999; Carrillo et al., 2001; Lötjönen and Mäkelä, 2001). In (Behloul et al., 2001), maximal myocardial deformation from tagged MRI and FDG-PET metabolism were combined using neuro-fuzzy rules to generate polar maps representing the viability. The registration was performed manually with the help of the long axis angles defined in the MRI protocol. In the "MunichHeart" software, endocardial and epicardial contours were manually delineated in Short Axis (SA) MR images and registered with the same contours extracted from PET or SPECT using the maximum count detection algorithm (Nekolla et al., 1998, 2000).

In this work, we propose a method for extracting an individualized 3-D anatomical heart model which combines myocardial metabolic data from PET and electrical measurements from MCG. A first approach of the method was presented in Mäkelä et al. (2001b). Here, it is improved by adding functional MCG results to the model. The method provides a 3 -D geometric representation of the heart onto which functional information can be displayed. The data are presented in section 2. An overview of the approach is given in section 3 , and the different steps are described in section 4 . The extraction of an individualized heart model is explained in section 5. The construction of functional cartographies are described in section 6 . Results are presented in section 7 and discussed in section 8 .

\section{Cardiac imaging protocol}

The cardiac data were composed of MR and PET images and MCG data of ten patients (identified by P1 to P10) suffering from three vessels coronary artery disease, diagnosed with coronary angiography and regional dyskinesia in cineangiograms (Lauerma et al., 2000). The mean age was 69 (8 men, 2 women). All patients underwent MR and fluorine-18-deoxyglucose (FDG) PET imaging within 10 days. MCG measurements were also acquired.

\section{- MR images}

The MR data were acquired with a $1.5 \mathrm{~T}$ Siemens Magnetom Vision imager (Siemens, Erlangen, Germany) at the Department of Radiology in Helsinki University Central Hospital (HUCH). A series of 39 ECG-gated contiguous transaxial images was acquired during free respiration using a TurboFLASH sequence (Fig. 2a). The pixel size and the slice thickness were $1.95 \times 1.95$ $\mathrm{mm}$ and $10 \mathrm{~mm}$, respectively. Five ECGgated breath-hold cine SA sections were also acquired (Fig. 2b). The pixel size for SA slices was $1.25 \times 1.25 \mathrm{~mm}$ and the slice thickness $7 \mathrm{~mm}$ with a gap of $15 \mathrm{~mm}$ between slices. About 15 time points were taken for each section with a repetition time of 40 msec.

\section{- PET images}

The static cardiac PET data were acquired with a Siemens ECAT 931/08-12 (Siemens/CTI, Knoxville, USA) PET scanner at the Turku PET Centre (Turku, Finland). A series of 16 contiguous transmission and emission images were acquired (Fig. 2c, 2d). Transmission images were used for attenuation correction of emission images and also provided structural information that was utilized for registration purposes. The emission image, which can be assumed to be in good registration with the transmission image (Kim et al., 1991), gives absolute quantification of glucosis uptake. For both transmission and emission images, the pixel size and the slice thickness were $2.41 \mathrm{~mm} \times 2.41 \mathrm{~mm}$ and $6.75 \mathrm{~mm}$, respectively. 


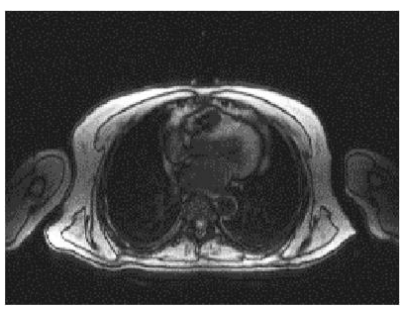

(a)

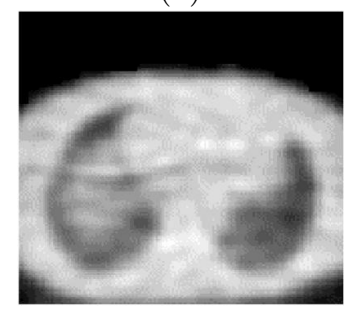

(c)

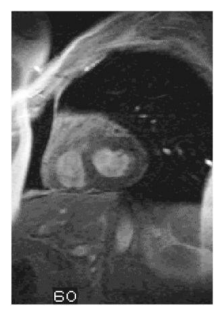

(b)

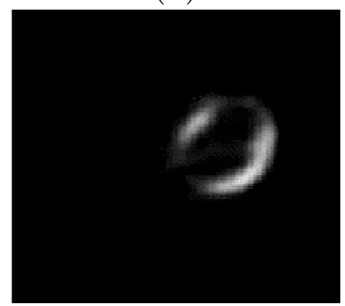

(d)
Figure 2. (a) Transaxial and (b) SA MR images, (c) transmission and (d) emission PET images.

\section{- MCG data}

The MCG measurements were performed at rest and after stress with the 67-channel cardiomagnetometer (4-D NeuroImaging, Helsinki, Finland) at the BioMag Laboratory (Montonen et al., 2000) at $\mathrm{HUCH}$. Acute ischemia was induced by exercise testing with a non-magnetic stress ergometer (Hänninen et al., 2001), pedaled in supine position.

The ST-segment difference signals of averaged post-stress and rest recordings were used in computing current density estimates (CDE) (Nenonen et al., 2001). In this work, the depolarization (QRS complex) data at rest was utilized. Patient-specific boundary-element torso models were acquired from magnetic resonance images, including the triangulated thorax and LV surfaces (Lötjönen et al., 1999; Pham et al., 2001). The torso was assigned a constant electrical conductivity of $0.2 \mathrm{~S} / \mathrm{m}$. Discrete CDE values were computed on the $\mathrm{LV}$ at midwall locations. The ill-posed inverse problem was regularized with three different methods (Tikhonov regularization with an identity or a surface Laplacian operator, and a maximum a posteriori estimator, MAP; for details, see Nenonen et al. (2001)). In the present study, we selected MCG results obtained with the MAP estimator.

\section{Method Overview}

The aim of the overall approach is to extract a 3 -D anatomical model of the heart from patient MR images and to incorporate functional data, such as FDG uptake, information of the magnetoelectric properties of the heart and other clinically relevant parameters to the model. The process is summarized in Fig. 3.

First, MR transaxial images were co-registered (rigid transformation) with the PET transmission image (Mäkelä et al., 2001a). The obtained registration parameters were used to register transaxial MR images and the PET emission image. PET slices that correspond to SA MR images were calculated by using MR header information. Then, a 3-D biventricular deformable model was initialized in the SA MR images to segment the myocardium. The deformed model was transformed into the registered SA PET image to obtain FDG uptake values. The model was also transformed to the transaxial MR image and MCG values were calculated for the LV midwall locations. The main steps of the method are described in the following sections.

\section{Registration}

\subsection{PET-MRI registration}

The rigid registration method for cardiac PET and MR images was fully described in (Mäkelä et al., 2001a). The registration method is based on the matching of the thorax and lungs surfaces which are visible in both PET transmission and MR transaxial images. The registration protocol first matches PET transmission and transaxial MR images by using a surface-based registration algorithm and then computes the SA PET slices corresponding to the SA MR slices. The 


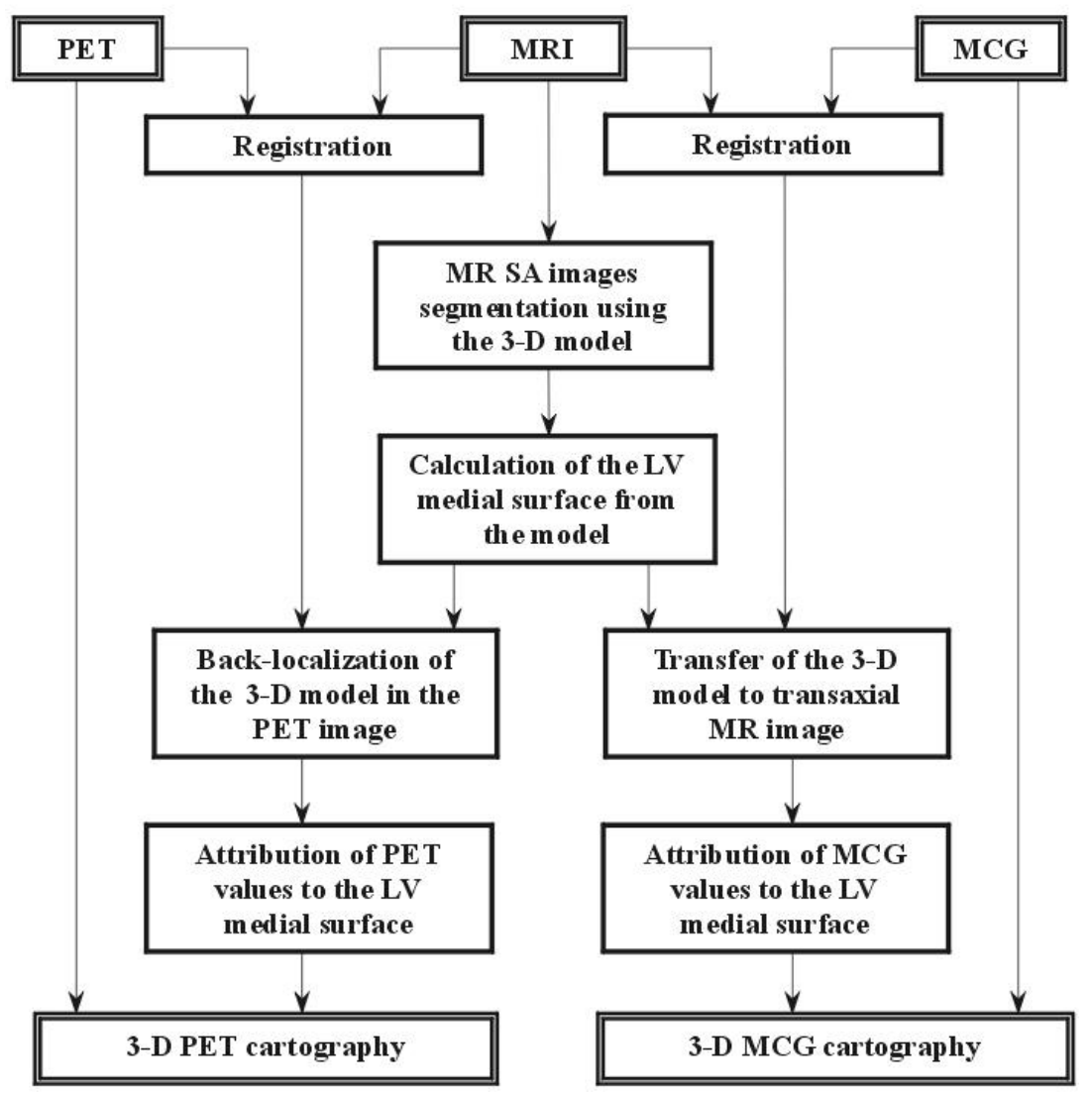

Figure 3. Extraction of the 3-D anatomical and functional model of the heart including PET-FDG uptake and MCG values: main steps of the process.

emission and transmission PET and transaxial MR images were first interpolated to the same isotropic voxel dimensions using tri-linear interpolation. Then, the PET transmission and emission images, which had smaller physical dimensions than MR image, was set to the geometric center of the MR image space. The thorax and lung surfaces were segmented from PET transmission image using the deformable model based segmentation method proposed in Lötjönen et al. (1999). Segmentation of the thorax structures (thorax and lungs surfaces) was based on the elastic deformation of a topologic and geometric prior model using a multiresolution approach. A thorax model including a full triangulated thorax and the lungs surfaces was used with MR images (Fig. 4a) while with the PET transmission images, the model was truncated (Fig. 4b). The initialization of the models can be made in three ways in our software: 1) manually, 2) matching bounding boxes set around the model and binarized (thresholded) volume or 3) making rigid registration with the model and binarized volume. In this work, the initialization was done manually. However, we have also used successfully the second approach in more than 50 cases as the thorax is segmented from MR images. Although the bounding boxes do not allow any rotation correction, it has performed well because subjects are always lying on the bed in an approximately 
same orientation. The segmentation algorithm can cope initialization errors up to a few degrees and several centimeters as demonstrated also in the original article (Lötjönen et al., 1999).

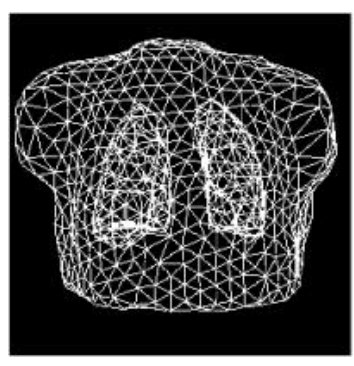

(a)

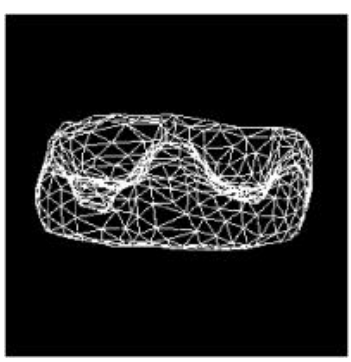

(b)
Figure 4. Geometric and topologic prior model of the thorax for the (a) MR and (b) PET transmission image segmentation.

The free-form based deformation algorithm adapts the prior model to locally fit the salient edges in the image within a minimization process. The energy to be minimized is

$E_{\text {total }}=E_{\text {image }}+\gamma E_{\text {model }}$,

where $E_{\text {image }}$ represents the matching error between the prior model and the partial edges in the data volume. $E_{\text {model }}$ tends to preserve the model's shape by restricting the deformation of the prior model. It describes the deviation of the model's surface normals from their original orientation. The image energy results from a distance map (Borgefors, 1988) built upon edges extracted either by a Canny-Deriche method (Canny, 1986) or image thresholding (Fig. 5). In order to select edges corresponding to the model, oriented distance maps (Lötjönen et al., 1999) were computed. The parameter $\gamma$ sets the contribution of the two energy components. A multiresolution process speeds up the minimization process and improves the convergence. Fig. 6a and Fig. 6b show segmentation results for the thorax structures in MR and PET transmission images.
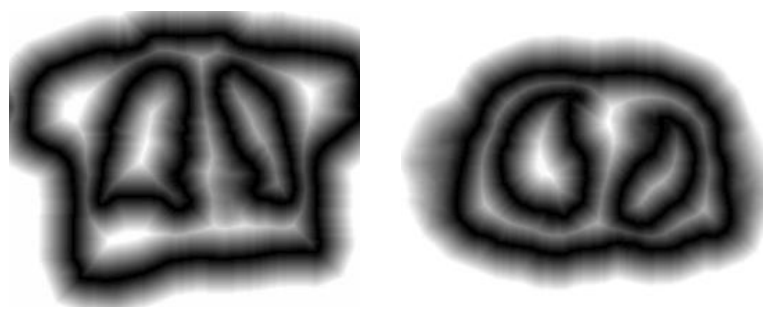

Figure 5. MR distance maps. The registration algorithm minimizes the distance of the thorax surface points in PET relatively to this distance map.

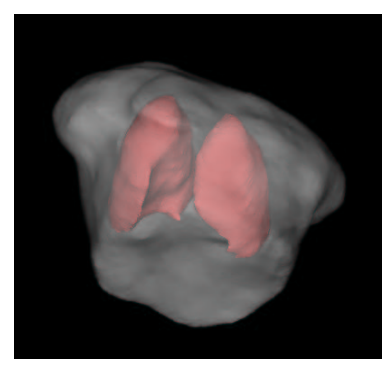

(a)

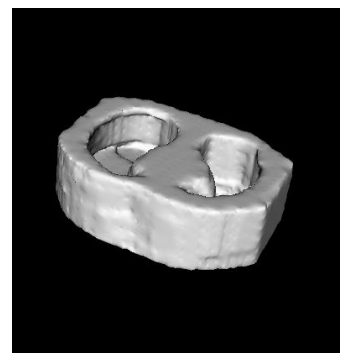

(b)
Figure 6. Segmented (a) MR and (b) PET transmission images.

The parameters of the rigid transformation parameters ( 3 translations, 3 rotations) between the two image sets are derived from the minimization of the distance between the point set from segmented PET transmission image surfaces and a distance map (Borgefors, 1988) built upon the segmented transaxial MR surfaces (Fig. 5). The uniformly distributed nodes of the deformable model were used as point set from PET transmission image surface. PET transmission and MR transaxial images are acquired in a similar geometry; the model is therefore initialized in a position close to the registration solution. The minimization algorithm is more detailed in (Mäkelä et al., 2001a). 
PET emission image was registered with transaxial MR image using the computed registration parameters. The PET transmission image was thus used as a linking mediator to register PET emission image to MR image coordinates.

After registration of the PET emission image with the transaxial MR image, the SA PET slices that corresponded to the SA MR images were computed using MR header information of transaxial and SA MR images (Fig. 7). Note that, as PET acquisition was not gated in this study, the PET images were registered with the SA MR data at end-diastole. This cardiac instant best corresponds to the average PET image.

\subsection{MCG-MRI registration}

MCG measurements were registered with MRI data by using external markers. The position of the MCG recording system with respect to the patient is determined by attaching three marker coils (magnetic dipoles) to the skin. The magnetic fields produced by the coils are then used to compute the sensor locations relatively to the marker coils (Montonen et al., 2000). The three marker coils were also used to define the MCG sensor coordinates with respect to the MCG markers, a set of nine external marker positions which were selected for registering the MCG sensor system to MR images (Fig. 8).

The locations of the MCG markers were defined by attaching a cross-shaped object consisting of two silicone strips of rubber on the skin of the thorax. The separation between neighboring MCG markers was $5 \mathrm{~cm}$ in the head-feet direction and $10 \mathrm{~cm}$ in the left-right direction. The locations of the MCG markers and the small marker coils were defined with a 3-D digitization system (3SPACE ISOTRAK II, Polhemus Inc., Colchester, VT, USA). The digitized MCG marker positions were stamped with non-toxic ink, visible only in ultraviolet light. Prior to MR imaging, the nine MRI markers were placed on the stamped positions on the skin. The MRI markers were constructed from two perpendicular tubes filled with $1 \mathrm{mmol} / \mathrm{l} \mathrm{MnCl}_{2}$ fluid, inserted inside a piece of plastic of $4.0 \times 4.0 \times 0.7 \mathrm{~cm}$. The cross-shaped figure of a marker was well visible in the MR images. The MRI markers were located manu-

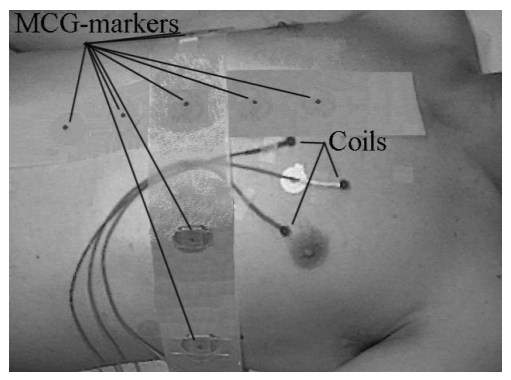

Figure 8. Placements of the nine MCG markers and three marker coils on the chest in a typical patient study. The round pieces of plastic in two silicone strips of rubber denote the MCG marker locations. Their centerpoints are digitized, and the pieces are removed from the strips before stamping the locations with ink visible in UV light. The three marker coils are used to define the MCG sensor coordinates in respect to the MCG markers.

ally from MR images, using a dedicated software. The nine marker coordinate sets $(x, y, z)$ in the MCG and MRI coordinate systems, respectively, were registered using a non-iterative least-squares method (Arun et al., 1987). Only rigid transformations including global rotations and translations were considered. MCG-MRI registration protocol has been applied to more than 50 patient studies (Pesola et al., 2000). With our patient studies we have obtained the root mean square (RMS) error of the nine registered markers to be about $6 \mathrm{~mm}$. The registration error includes various error sources such as the effect of breathing, reattachment of the markers between MCG and MR measurements, localization of the markers from MR images and shape changes in thorax between the measurements because of the flexibility of the shoulders and the skin (Mäkelä et al., $2002 b$ ). One of the reasons why the RMS error values are relatively low in our measurements is that the markers in our protocol were attached in positions which were not very sensitive to different alignment errors. 


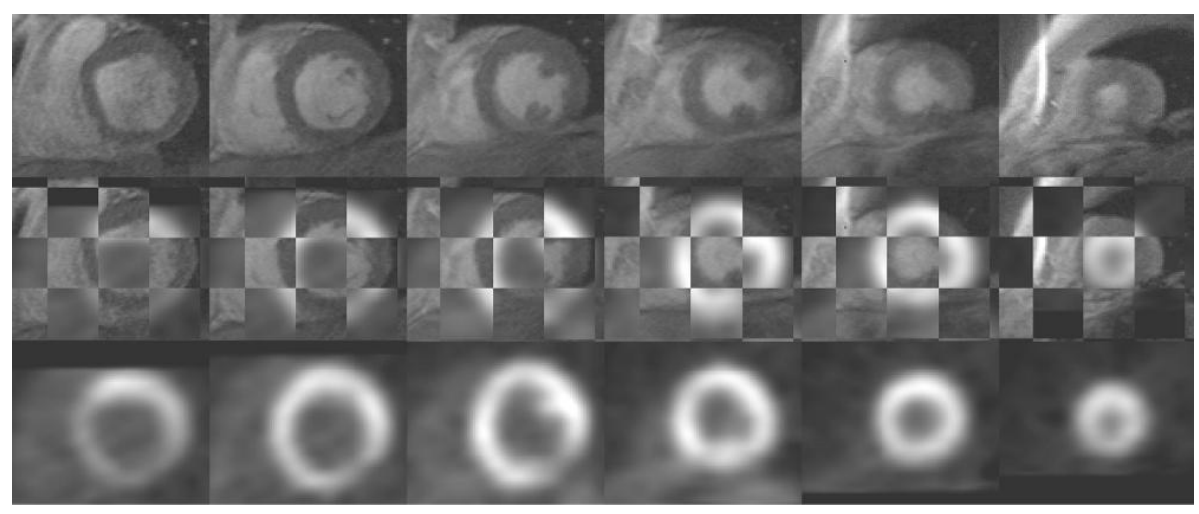

Figure 7. A stack of registered end-diastolic SA MR (top) and PET emission (bottom) image slices for the $\mathrm{P} 1$ case. In the middle row, the images were overlaid in block format to visualize registration results.

\section{Capturing the heart anatomy}

MR images provide relevant information on the cardiac anatomy. In this section, we present a method for extracting a 3-D individualized anatomical model of the heart from MR short axis images, based on an Elastic Active Region Model (ARM) which is an extension of our previous work (Pham et al., 2001). Several groups have proposed superficial deformable models to segment the cardiac chambers in three dimensions (McInerney and Terzopoulos, 1996). More recently, Montagnat and Delingette (2000) introduced simplex meshes for segmenting the LV surfaces from 4-D cardiac sequences in MR, SPECT and ultrasound imaging. The reader can find in (Frangi et al., 2001) a good review of 3-D heart modeling approaches used for the purpose of segmentation. Contrary to superficial models, the ARM relies on a volumetric deformation of a geometric heart model. It allows to simultaneously extract the LV and RV endocardial surfaces, as well as the epicardium, while enforcing regularity between these surfaces. In addition, it is formulated in the mechanical framework of elastic bodies that is often used in the context of motion estimation (Papademetris et al., 2001). The ARM is also closely related to the work of Sermesant et al. (2001) who introduced an electromechanical model of the heart. In this model, the contraction is controlled by simulating the propagation of electrical waves and taking into account the interaction with ultrasound images. Nevertheless, in this work, the objective is not to provide a complete biomechanical model of the heart, but to accurately segment MR cardiac images by means of a simple but natural description of the heart. The segmentation process can be divided in two main steps. First, the template is spatially positioned by registering it with the image to be segmented. Then, starting from this initial configuration, it is elastically deformed to fit the cardiac structures. Each step will be described in the following.

\subsection{The 3-D biventricular template}

A priori information on the shape of the object of interest strongly constrains the segmentation. We thus developed a $3-D$ geometric accurate representation of the heart ventricles. This volumetric template was created from a MR reference dataset. The heart of a healthy volunteer was imaged using a cine-MR protocol and the enddiastolic time frame was selected, as we only focus on the images corresponding to the end-diastole. A medical expert interactively delineated the epiand endocardial contours of the two ventricles on each of the 2-D short axis slices. The corresponding surfaces were then reconstructed and the interior meshed with tetrahedra using the GHS3D 
software (INRIA, Gamma Project, France). The 3-D biventricular template is shown in Fig. 9.

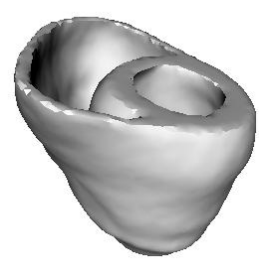

(a)

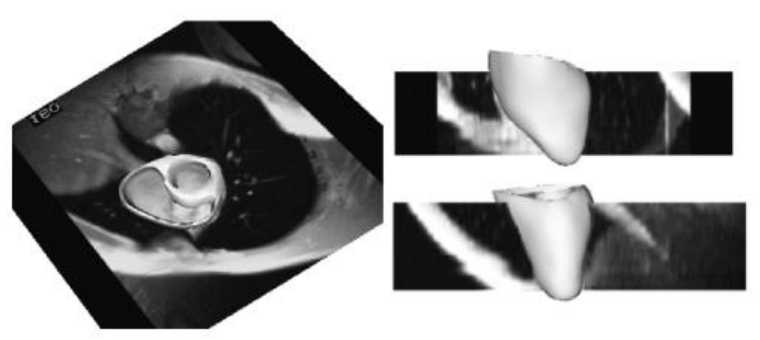

(b)

Figure 9. (a) 3-D prior biventricular model (19524 nodes, 94402 tetrahedral elements), (b) model immersed in the MR data.

\subsection{Interpolating 3-D MR volumes}

In order to take advantage of a fully threedimensional interaction between the model and the short axis images of the heart, 3-D isotropic volumes were interpolated from stacks of $2-\mathrm{D}$ short axis slices. SA images present a good spatial resolution in the acquisition plane, but a poor one in the transversal direction. For the studied cases, the inter-slice distance was $15 \mathrm{~mm}$, whereas the pixel size was less than $1.5 \mathrm{~mm}$. In such conditions, we experimentally observed that the shape based interpolation technique (Grevera and Udupa, 1996) gave better results than polynomial interpolations on our data.

\subsection{The model initialization as a registra- tion step}

The model is rigidly positioned with respect to the image to be as close as possible to the cardiac structures to be segmented. This is done by searching for the 6 parameters of the rigid spatial transformation $T$ (3 translations, 3 rotations) applied to the model, that minimizes the following energy :

$E_{i n i}(T)=E_{\partial \Omega}(T) E_{W}(T)$

Like in (Pluim et al., 2000), the energy to be minimized is a product of an energy based on an intensity criterion and an energy deriving from the image gradient. Here, $E_{\partial \Omega}(T)$, computed on the boundary of the template, represents a distance from the model to detected contours in the image. In practice, we take the mean square Euclidean distance from the boundary nodes to the image edges. The region energy $E_{W}(T)$ is a similarity measure between the image intensities. It is estimated in a floating meshed domain $W$ surrounding the template $(\Omega \subset W)$ and can either be the sum of the square intensity differences (SSD) sampled on the nodes of $W$, or the mutual information (MI) between the two gray level distributions. In most of the cases, good results are obtained with the SSD criterion which is known to be efficient in the monomodal case (Fitzpatrick et al., 2000). The total initialization energy is minimized using the Powell's optimization method (Press et al., 1988) which does not require the computation of the function's gradient. Fig. 10 shows initialization results for two patients.

\subsection{The deformation model}

The second step of the segmentation process is seen as the deformation of an elastic body submitted to an external force field. The myocardium is modeled as a linear elastic continuous medium. Under the small deformation assumption, the linearized form of the Green-Lagrange strain tensor is used:

$[\boldsymbol{\epsilon}]=\frac{1}{2}\left(\nabla \mathbf{u}+\nabla \mathbf{u}^{\mathbf{t}}\right)$

where $\mathbf{u}$ denotes the displacement vector. The stress is related to the strain by the constitutive 

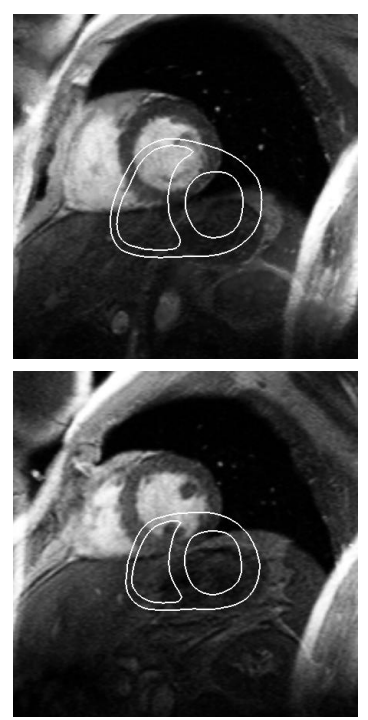

(a)
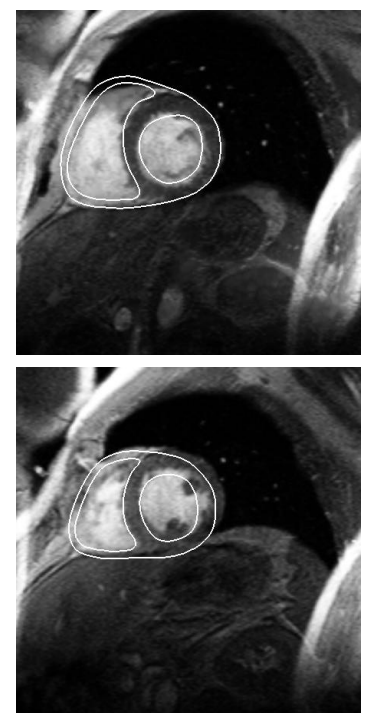

(b)
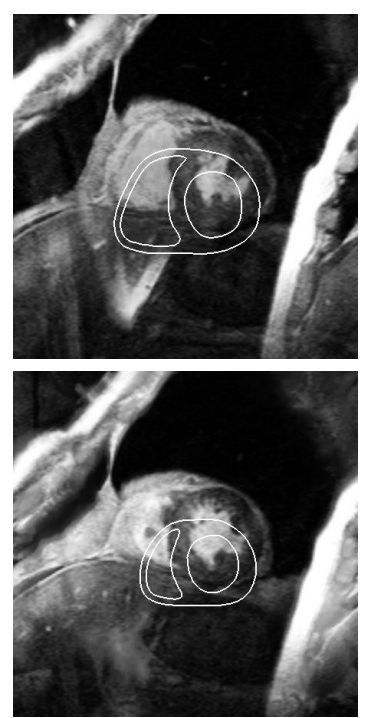

(c)
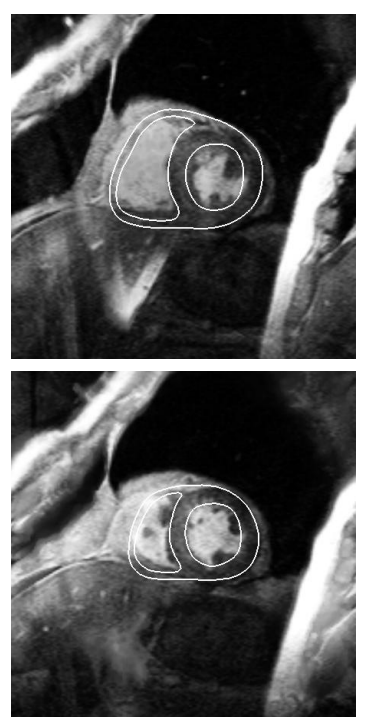

(d)

Figure 10. Initialization step. (a) : patient P1, before registration, (b) : patient P1, after registration, (c) : patient P2, before registration, (d) : patient P2, after registration. First row : basal cross-section, second row : mid-ventricular level.

law of the material. For a homogeneous isotropic material, it takes the form:

$\boldsymbol{\sigma}=\mathbf{R} \varepsilon=\mathbf{R S u}$

where $\quad \boldsymbol{\sigma}=\left(\sigma_{11}, \sigma_{22}, \sigma_{33}, \sigma_{12}, \sigma_{23}, \sigma_{31}\right)^{t} \quad$ and $\varepsilon=\left(\varepsilon_{11}, \varepsilon_{22}, \varepsilon_{33}, \varepsilon_{12}, \varepsilon_{23}, \varepsilon_{31}\right)^{t}$ are respectively the stress and the strain vectors, $\mathbf{R}$ the elasticity matrix depending on two mechanical parameters (either the Lamé coefficients $\lambda$ and $\mu$, or the Young modulus $E$ and the Poisson ratio $\nu$ ), and $\mathbf{S}$ a differential operator. If we note $\Omega$ the considered domain, $\partial \Omega$ its boundary, and $\mathbf{t}$ the superficial external forces applied on the boundary, the equilibrium state corresponds to the minimum of the following potential energy :

$$
E_{\text {glob }}(\mathbf{u})=\underbrace{\frac{1}{2} \int_{\Omega} \boldsymbol{\sigma} \varepsilon d \mathbf{x}}_{E_{\text {elast }}(\mathbf{u})}-\alpha \underbrace{\int_{\partial \Omega} \mathbf{t}(\mathbf{I}+\mathbf{u}) \cdot \mathbf{u} d \mathbf{s}}_{E_{\text {data }}(\mathbf{u})}(5)
$$

The first term $E_{\text {elast }}(\mathbf{u})$ is an elastic energy which regularizes the deformations imposed by the external energy $E_{\text {data }}(\mathbf{u})$, evaluated in the deformed configuration (if we assume that the displacements are small). In the case of large displacements, the geometric non-linearity should be taken into account by updating the elastic energy during the deformation process. The external force field deriving from the image is chosen such that it tends to attract the model's boundary towards salient features existing in the image. One way to compute a $3-\mathrm{D}$ force field is to calculate the gradient of a potential image $\mathbf{t}(\mathbf{x})=-\nabla P(\mathbf{x})$ which can be, for example, either the norm of the image gradient, an edge map extracted with a Canny-Deriche operator and smoothed with a gaussian filter, or a distance map (chamfer or Euclidean distance). The Gradient Vector Flow (GVF), proposed by $\mathrm{Xu}$ and Prince (1998), directly provides a force field which allows a better convergence, especially in the case of concavities. In our experiments, the GVF computed from the norm of the image gradient was used in most of the cases.

Eq. (5) is discretized using the Finite Element Method (FEM) with linear basis functions 
(Zienkiewicz and Taylor, 1987). The energy to be minimized thus becomes :

$E_{g l o b}(\mathbf{U})=\frac{1}{2} \mathbf{U}^{t} \mathbf{K U}+\alpha \mathbf{F} \cdot \mathbf{U}$

with $\mathbf{U}$ being the global displacement vector, $\mathbf{F}$ the global force vector and $\mathbf{K}$ the stiffness matrix. A minimum is reached for :

$\mathbf{K U}=\mathbf{F}$

As $\mathbf{F}$ is a function of the displacement, Eq. (7) is solved iteratively using a semi-implicit Euler scheme. Final segmentation results after the model deformation are shown in Fig. 11 for the two same cases.
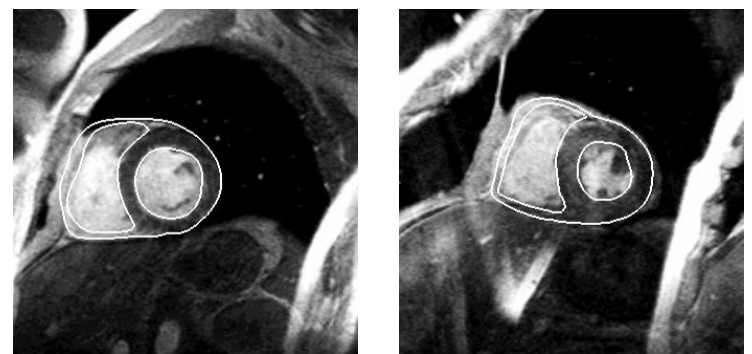

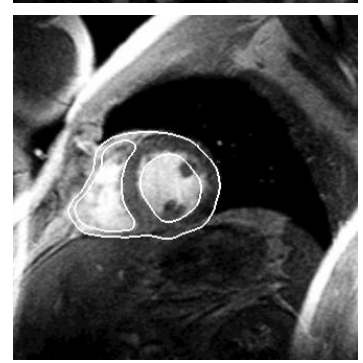

(a)

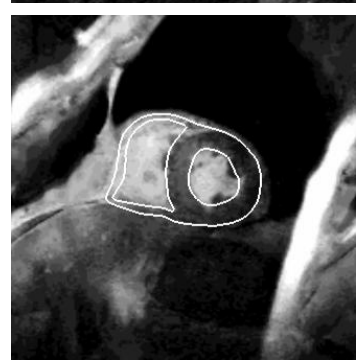

(b)
Figure 11. Segmentation results. (a) patient P1, (b) patient P2. First row : basal slice, second row : mid-ventricular level.

\section{Functional model of the heart}

\subsection{FDG-PET cartography}

After the registration and segmentation steps, functional data was attributed to the elements and nodes of the 3-D biventricular model. Also some parameters with clinical interest was derived from MR imaging. First, the model was labeled so that it was possible to separate the right and left ventricles and obtain internal and external surfaces. Moreover, cavity volumes, myocardial mass and local wall thickness were computed. In order to enrich the model with metabolic information, the model was transformed into the registered PET-FDG emission image. A LV medial surface was automatically calculated between LV endo- and epicardial surfaces (Fig. 12).

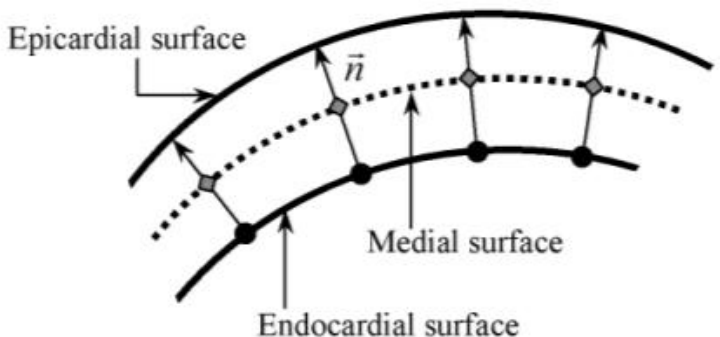

Figure 12. Calculation of the medial surface using the segmented epicardial and endocardial surfaces.

The medial surface nodes were calculated by, first computing the 3-D normal to each node of the endocardial surface and, secondly, calculating the middle point of the segment normal to the endocardial surface and its intersection with the epicardial surface (Fig. 13a). Surfaces were transformed to registered PET emission image and a FDG uptake mean value was computed at each node of the medial surface (Fig. 13b, middle contour) in a $5 \times 5 \times 5$ neighborhood, which corresponds to $6.25 \mathrm{~mm} \times 6.25 \mathrm{~mm} \times 6.25 \mathrm{~mm}$ physical dimensions (Fig. 13c).

\subsection{Magneto-electric cartography}

MCG inverse current-density estimates were calculated for the nodepoints of the medial surface. To this aim, the medial surface was transformed from MR SA plane to transaxial MR co- 


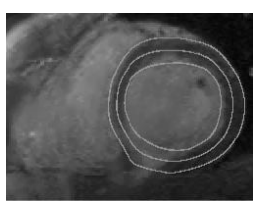

(a)

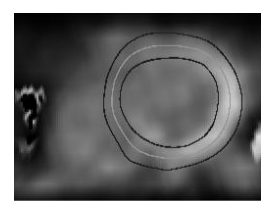

(b)

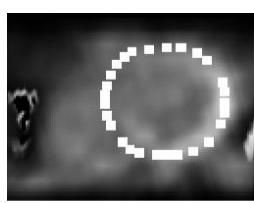

(c)

Figure 13. (a) Intersection of endocardial (inner contour), epicardial (outermost contour) and calculated medial (middle contour) surfaces with a MR SA slice, (b) the same contours are shown in the corresponding registered PET SA image (medial contour in the middle) and (c) the mean value of the FDG-uptake was computed at the medial surface nodes in a small neighborhood.

ordinate system by using MR header information. The MCG measurements were regularized with a MAP estimator.

\section{Results}

\subsection{FDG-PET cartography}

Fig. 14(left) and Fig. 15(left) illustrate the FDG metabolic activity over the LV medial surface for 2 cases. Right ventricular and epicardial surfaces are shown in transparency. In Fig. 14(right) and Fig. 15(right), the corresponding PET bull's eye (polarmap) presentations are shown for a qualitative evaluation of the 3-D displays (Siemens software, Turku PET Centre). Bull's eye images are commonly used method for displaying functional information of the heart. The center corresponds to the apex and the outermost ring represents the basal slice. In such polarmaps, metabolic activity is estimated at leftventricular mid-wall.

The corresponding low LV FDG uptake areas can clearly be seen in both the bull's eye images (dark green in Fig. 14-15(right)) and in the 3-D model displays (arrows in Fig. 14-15(left)). Visualizations of the 3-D model were performed using the VTK software library ${ }^{2}$.

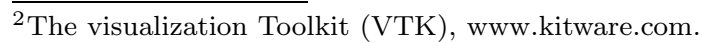

\subsection{Magneto-electric cartography}

MCG results are presented on the medial surface for P1 in Fig. 16 and P2 in Fig. 17. For both cases, the 3 -D visualization is presented on the left and the corresponding bull's eye representation computed from the extracted 3-D medial surface on the right. The MCG results were computed from the resting state data at a single time instant in the middle of the QRS complex. The results have to be regarded illustrative in that sense that the electrical activity in the right ventricle was neglected in the inverse calculations.

\section{Discussion and Conclusion}

In this paper, we presented a new approach for the combination of anatomical and functional data from various cardiac imaging modalities. It relies on the registration of thorax structures (thorax surfaces) which are extracted from MR anatomical and PET transmission images. In order to provide 3-D displays of the heart that are easily interpretable by the physician, an individualized 3-D biventricular heart model is extracted from the SA MR images. The various geometrical and functional parameters can therefore be represented onto this model through the LV medial surface (Fig. 14-17). Following our previous efforts, we show in this paper the ability of the method to integrate information about the magneto-electric activity of the heart from MCG. The fusion of metabolic data from PET and magneto-electrical potential onto the same individualized 3-D heart model from MRI has been illustrated on two pathological cases. With such an approach, the localization of metabolic and conduction defects is straightforward since the biventricular model allows for the unambiguous identification of myocardial territories. Within the same framework, it is natural to envisage the inclusion of other complementary functional data such as information related to the myocardial deformation or perfusion (Behloul et al., 2001).

The reliability of the method mainly depends on the accuracy of the rigid PET-MRI registration. The registration results of 10 cases were visually inspected by a medical expert. Nine over the 10 available cases were considered to provide 


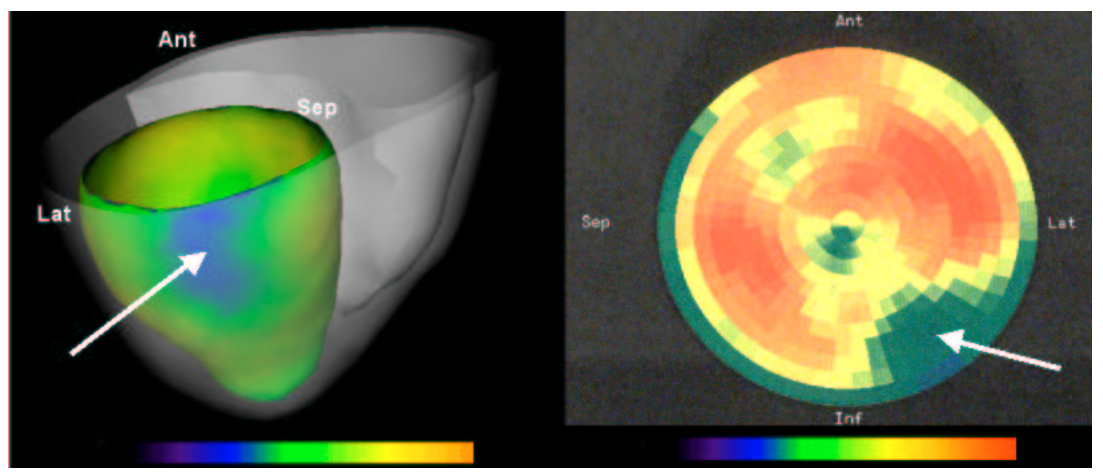

Figure 14. 3-D representation of the FDG PET uptake values on the biventricular heart model (left) for patient P1. Interactively made bull's eye representation (Siemens software) of the corresponding PET image is presented on the right. The polarmap shows PET values at mid-wall location. Scar area can be seen in red at the basal level of the 3 -D display (arrows).

satisfactory correspondence results. In the remaining bad case, unexpected artifacts were observed in FDG-PET data. In a previous paper (Mäkelä et al., 2001a), we proposed a quantification of the registration accuracy based on the calculation of a distance between segmented surfaces in PET and MRI. The mean distance was $3 \mathrm{~mm}$ with a maximum of $4 \mathrm{~mm}$. This is only an indication of the performance of the registration algorithm since it highly depends upon the segmentation of the thorax structures. It also provides a simple mean to detect possible registration failure. A more systematic and accurate validation of the registration method is currently conducted through image simulations. Also intensity based methods, like mutual information, are potential alternative to determine registration parameters in this kind of registration methods where the PET transmission image is used as a linking mediator to register PET emission image to MR image coordinates. In some cases, intensity based methods can even allow to register the emission image directly with the anatomical image.

PET imaging devices have typically 4 - $10 \mathrm{~mm}$ spatial resolution (Hartiala and Knuuti, 1995). In general, the ability of our 3-D model based method to detect functional abnormalities is lim- ited by the spatial resolution of the PET imaging device. In MCG, accuracies of 5 to $25 \mathrm{~mm}$ have been reported by comparing the MCG localization results to: i) cardiac surgery, ii) catheter ablation, iii) the results of invasive electrophysiological studies, iv) ECG localization results, and v) physiological knowledge (X-ray or MRI). Besides patient studies, the ability of the MCG to locate artificial current dipoles has been tested with physical thorax phantoms. A non-magnetic pacing catheter in a realistically shaped thorax phantom resulted in equal accuracies of $5-10 \mathrm{~mm}$ between MCG and ECG mapping data (Fenici et al., 1998). On the other hand, the same catheter in 15 patients showed significantly better localization accuracy with MCG data $(7 \mathrm{~mm})$ than with simultaneously recorded ECG mapping data (25 mm) (Pesola et al., 1999).

The MCG results in this work were evaluated on the median LV surface during the cardiac depolarization. The resting-state MCG data in the peak of the QRS contains contributions from both ventricles, while the $\mathrm{LV}$ contribution dominates. In this study the RV was not included in the inverse MCG computations. The main motivation to use only the LV surface came from our previous study where we investigated the MCG data after physical exercise test in the same patient popu- 


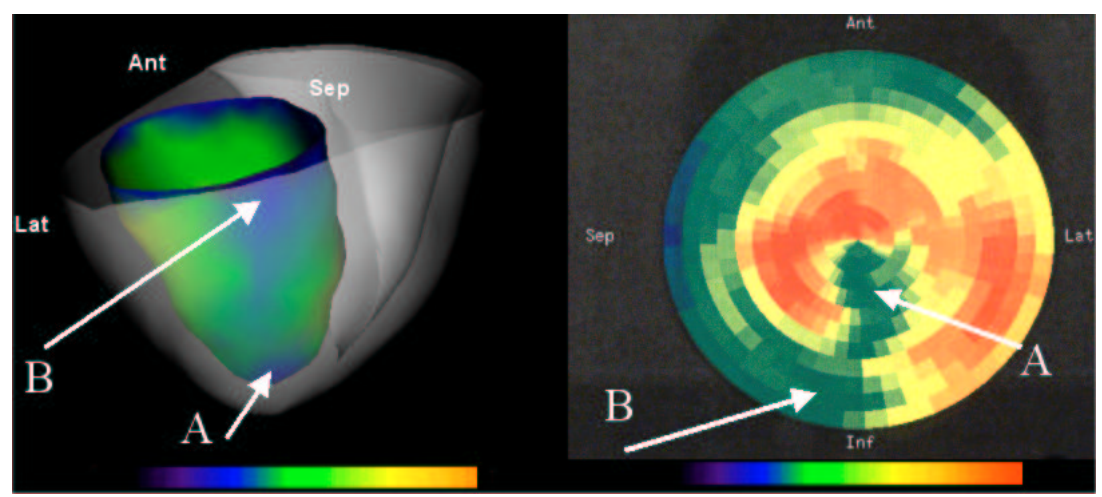

Figure 15. Left : 3-D representation of the FDG PET uptake values on the biventricular heart model for patient P2. Right : corresponding bull's eye representation (Siemens software) with values estimated at mid-wall location.

lation (Nenonen et al., 2001). In that study the ST segment was analyzed and difference signals were computed (post-exercise minus rest). This difference was associated with exercise-induced ischemia which was known to arise in the left ventricle on the basis of PET and MRI (Lauerma et al., 2000). While the whole MCG (and ECG) source imaging methodology is still under rapid development (Hämäläinen and Nenonen, 1999) the results show that MCG, due to a millisecond time resolution, may be able to provide new valuable information on cardiac function, especially when constrained with accurate anatomy from MRI and combined with other medical imaging data.

The temporal correspondence of the PET and MR images has been overcome by assuming the correspondence between the MR transaxial, enddiastolic SA images, and the PET acquisitions, as it is usually admitted in clinical practice. In fact, transaxial MR images were obtained with a snapshot technique during free respiration while PET measurements were accumulated during a relatively long period of time (about 10 minutes). This might generate differences in the thorax and lungs shape and, consequently, cause registration errors. ECG-gated PET with non-rigid registration could certainly help to better handle the temporal correspondence problem.
The results of the automatic extraction of the biventricular model using the ARM were quite satisfactory for the two processed cases as shown in Fig. 11. This step, which is independent of the others, is important since i) it extracts an individualized anatomical model from which volumes, masses, wall thickness can be derived, ii) it provides the region of interest where the functional parameters from PET and MCG are estimated. We are currently quantifying the performance of the ARM in terms of accuracy (as compared to manual tracing) and robustness, especially as a function of model initialization. In addition, acquisition sequences such as stimulated echo sequences (e.g TrueFISP, bFFE, Fiesta) provide better resolution and contrast and may improve the reliability and the accuracy of the segmentation results.

The medial surface seems to us the most straightforward way to obtain functional cartographies preventing from border effects that could occur from slight mis-registration. In this work, we implemented a simple geometrical approach to compute this surface. Other methods such as the centersurface (Bolson et al., 1995) could also be applied. In order to keep the volumetric property of the model, the next step will consist in the anatomical re-parameterization of the heart's geometry so that the functional pa- 


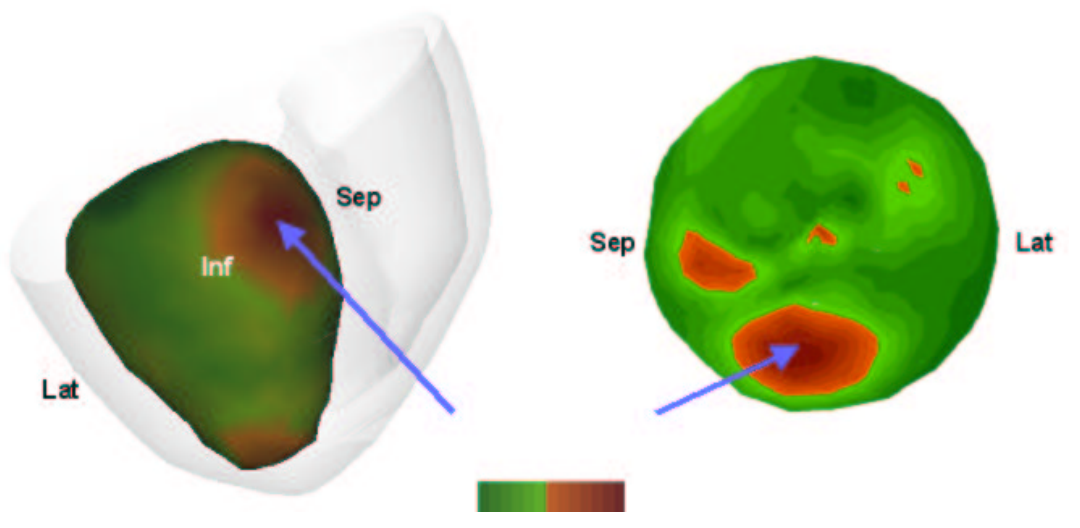

Figure 16. Left : 3-D representation of the MCG values on the biventricular heart model for patient P1. Right : interactively made corresponding bull's eye representation calculated from the 3-D medial surface. Scar area can be seen in red at the basal level of the presentations (arrows).

rameters can be easily depicted on the myocardial borders as well as inside the wall by interactively peeling the model, for instance.

In conclusion, we have presented a modelbased approach for the combination onto patientspecific heart model of both anatomical and diverse functional data from multimodality cardiac imaging. In this work, one of the main purposes was to automatically register metabolic PET and electromagnetic measurements from MCG. Similar approach could also be used to combine other modalities such as SPECT and multichannel ECG studies. We believe that the presented approach can help the radiologist or cardiologist to rapidly apprehend the functional state of the myocardium through 3-D intuitive cartographies. It is therefore possible to accurately compare the different imaged functions (metabolism, magneto-electric activity) in the context of physio-pathological studies on the ischemic diseases, for instance, which consequences on the cardiac muscle are complex and not yet fully understood. In order to enrich the model, other parameters related to the heart motion and perfusion will be integrated in the near future.

\section{Acknowledgments}

This study was partly granted by the Scientific Department of the French Embassy in Finland, the Region Rhône Alpes, through the ADéMO project and The Foundation of Technology in Finland. This work was performed within the framework of the joint incentive action "Beating Heart" of the research groups ISIS, ALP and MSPC of the French National Center for Scientific Research (CNRS).

\section{References}

Andersson, J. L. R., Bagnhammar, B., Schneider, H., 1995. Accurate attenuation correction despite movement during PET imaging. J. Nucl. Med. 36 (4), 670-678.

Arun, K. S., Huang, T. S., Blostein, S. D., 1987. Least-squares fitting of two 3-D point sets. IEEE Trans. Pattern Anal. Machine Intell. 9 (5), 698-700.

Behloul, F., Lelieveldt, B. P. F., Boudraa, A., Janier, M., Revel, D., Reiber, J. H. C., December 2001. Neuro-fuzzy systems for computeraided myocardial viability assessment. IEEE Trans. Med. Imaging 20 (12), 1302-1313. 


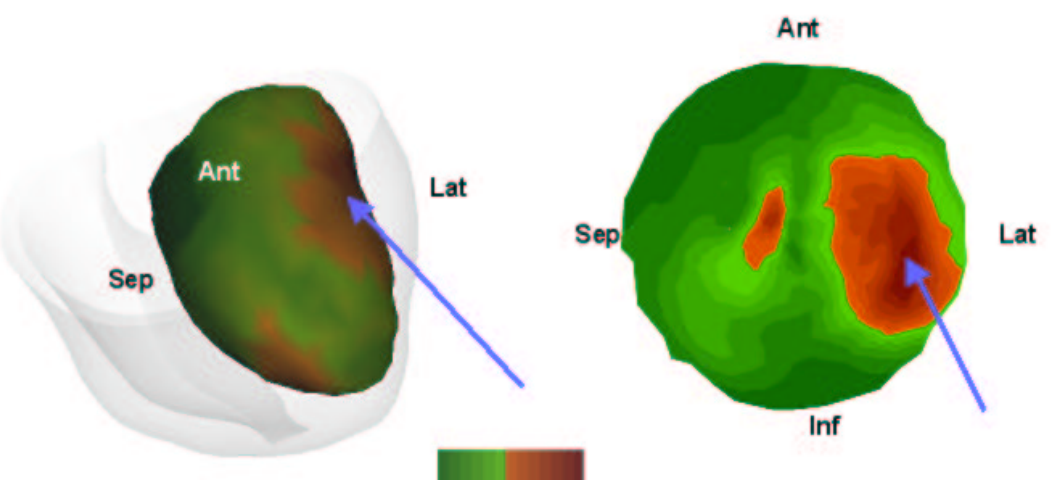

Figure 17. Left : 3-D representation of the MCG values on the biventricular heart model for patient P2. Right : interactively made corresponding bull's eye representation calculated from the 3-D medial surface.

Bettinardi, V., Gilardi, M., Lucignani, G., Landoni, C., Rizzo, G., 1993. A procedure for patient repositioning and compensation for misalignment between transmission and emission data in PET heart studies. J. Nucl. Med. 34 (1), 137-142.

Bolson, E., Sheehan, F., Legget, M., Jin, H., McDonald, J., Sampson, P., Martin, R., Bashein, G., Otto, C., 1995. Applying the centersurface model to $3-\mathrm{D}$ reconstructions of the left ventricle for regional function analysis. In: Computers in Cardiology, IEEE Computer Society. Long Beach, CA, pp. pp. 63-66.

Borgefors, G., 1988. Hierarchical chamfer matching: A parametric edge matching algorithm. IEEE Trans. Pattern Anal. Machine Intell. 10 (6), 849-865.

Cai, J., Chu, J., Recine, D., Sharma, M., Nguyen, C., Rodebaugh, R., Saxena, A., Ali, A., 1999. $\mathrm{CT}$ and PET lung image registration and fusion in radiotherapy treatment planning using the chamfer-matching method. Int. J. Radiation Oncology Biol. Phys. 43 (4), 883-891.

Canny, J., November 1986. A computational approach to edge detection. IEEE Trans. Pattern Anal. Machine Intell. 8 (6), 679-698.
Carrillo, A., Duerk, J., Lewin, J., Wilson, D., 2001. Semiautomatic 3-D image registration as applied to interventional MRI liver cancer treatment. IEEE Trans. Med. Imaging 19 (3), $175-185$.

Declerck, J., Feldmar, J., Goris, M., Betting, F., 1997. Automatic registration and alignment on a template of cardiac stress and rest reoriented SPECT images. IEEE Trans. Med. Imaging 16 (6), 727-737.

Dey, D., Slomka, P., Hahn, L., Kloiber, R., 1999. Automatic three-dimensional multimodality registration using radionuclide transmission CT attenuation maps: A phantom study. J. Nucl. Med. 40 (3), 448-455.

Eberl, S., Kanno, I., Fulton, R., Ryan, A., Hutton, B., Fulham, M., 1996. Automated interstudy image registration technique for SPECT and PET. J. Nucl. Med. 37 (1), 137-145.

Faber, T., McColl, R., Opperman, R., Corbett, J., Peshock, R., 1991. Spatial and temporal registration of cardiac SPECT and MR images: methods and evaluation. Radiology 179 (3), 857-861.

Fenici, R., Nenonen, J., Pesola, K., Korhonen, P., Lötjönen, J., Mäkijärvi, M., Poutanen, V. P., 
Keto, P., Katila, T., 1998. Non-fluoroscopic localization of an amagnetic stimulation catheter by multichannel magnetocardiography. PACE $22,1210-1220$.

Fitzpatrick, J., Hill, D., Maurer, C., 2000. Handbook of Medical Imaging. Vol. 2. SPIE Press, Ch. Image registration, pp. 375-435.

Frangi, A. F., Niessen, W. J., Viergever, M. A., 2001. Three-dimensional modeling for functional analysis of cardiac images, a review. IEEE Trans. Med. Imaging 20 (1), 2-25.

Gilardi, M. C., Rizzo, G., Savi, A., Landoni, C., Bettinardi, V., Rossetti, C., Striano, G., Fazio, F. N., 1998. Correlation of SPECT and PET cardiac images by a surface matching registration technique. Computerized Medical Imaging and graphics $22,391-398$.

Grevera, G. J., Udupa, J. K., December 1996. Shape-based interpolation of multidimensional grey-level images. IEEE Trans. Med. Imaging 15 (6), 881-892.

Hämäläinen, M., Nenonen, J., 1999. Encyclopedia of Electrical Engineering. Vol. 12. New York: Wiley \& Sons, Ch. Magnetic Source Imaging, pp. 464-479.

Hänninen, H., Takala, P., Mäkijärvi, M., Montonen, J., Korhonen, P., Oikarinen, L., Simelius, K., Nenonen, J., Katila, T., Toivonen, L., 2001. Recording locations in multichannel magnetocardiography and body surface potential mapping sensitive for regional exercise-induced ischemia. Basic Res. Cardiol. 96, 405-414.

Hartiala, J., Knuuti, J., 1995. Imaging of heart by MRI and PET. Ann. Med. 27, 35-45.

Hoh, C., Dahlbom, M., Harris, G., Choi, Y., Hawkins, R., Philps, M., Maddahi, J., 1993. Automated iterative three-dimensional registration of positron emission tomography images. J. Nucl. Med. 34 (11), 2009-2018.

Kim, R., Aw, T., Bacharach, S., Bonow, R., 1991. Correlation of cardiac MRI and PET images using lung cavities as landmarks. In: Proc.
IEEE Conf. Computers in Cardiology. pp. 49 52.

Lauerma, K., Niemi, P., Hänninen, H., Janatuinen, T., Voipio-Pulkki, L., Knuuti, J., Toivonen, L., Mäkelä, T., Mäkijärvi, M., Aronen, H., 2000. Multimodality MR imaging assessment of myocardial viability, combination of first-pass and late contrast enhancement to wall motion dynamics and comparison with FDG-PET. Radiology 217, 729-736.

Lötjönen, J., Mäkelä, T., 2001. Elastic matching using a deformation sphere. In: Niessen, W., Viergever, M. (Eds.), Lecture Notes in Computer Science 2208: Medical Image Computing and Computer-Assisted Intervention, MICCAI01. Springer, pp. 541-548.

Lötjönen, J., Reissman, P.-J., Magnin, I. E., Katila, T., 1999. Model extraction from magnetic resonance volume data using the deformable pyramid. Med. Image Anal. 3 (4), $387-406$.

MacLeod, R. S., Brooks, D. H., January 1998. Recent progress in inverse problems in electrocardiology. IEEE Engineering in Medicine and Biology 17 (1), 73-82.

Mäkelä, T., Clarysse, P., Lötjönen, J., Sipilä, O., Lauerma, K., Hänninen, H., Nenonen, J., Knuuti, J., Katila, T., Magnin., I. E., 2001a. Understanding cardiac imaging techniques from basic pathology to image fusion. Vol. 322 . IOS Press, Ch. A Method for Registration of Cardiac Magnetic Resonance and Positron Emission Tomography Images for Assessing Myocardial Viability, pp. 155-165.

Mäkelä, T., Clarysse, P., Lötjönen, J., Sipilä, O., Lauerma, K., Hänninen, H., Pyökkimies, E.-P., Nenonen, J., Knuuti, J., Katila, T., Magnin, I. E., 2001b. A new method for the registration of cardiac $\mathrm{PET}$ and $\mathrm{MR}$ images using deformable model based segmentation of the main thorax structures. In: Medical Image Computing and Computer-Assisted Intervention (MICCAI'01). Vol. 2208 of Lecture Notes 
in Computer Science (LNCS). Springer, pp. $557-564$.

Mäkelä, T., Clarysse, P., Sipilä, O., Pauna, N., Pham, Q., , Katila, T., Magnin, I. E., September 2002a. A review of cardiac image registration methods. IEEE Trans. Med. Imaging 21 (9), to appear.

Mäkelä, T., Lötjönen, J., Sipilä, O., Lauerma, K., Nenonen, J., , Katila, T., Magnin, I. E., 2002b. Error analysis of registering of anatomical and functional cardiac data using external markers. In: Nowak, H., Haueisen, J., Giesler, F., Huonker, R. (Eds.), Biomag 2002, Proc. of the 13th Internation. Conf. on Biomagnetism. pp. $842-845$.

McInerney, T., Terzopoulos, D., 1996. Deformable models in medical image analysis: a survey. Med. Image Anal. 1 (2), 91-108.

Montagnat, J., Delingette, H., 2000. Space and time shape constrained deformable surfaces for 4D medical image segmentation. In: Medical Image Computing and Computer-Assisted Intervention (MICCAI'00). Vol. 1935 of Lecture Notes in Computer Science (LNCS). Springer, pp. 196-205.

Montonen, J., Ahonen, A., Hämäläinen, M., Ilmoniemi, R., Laine, P., Nenonen, J., Paavola, M., K. Simelius, K., Simola, J., Katila, T., 2000. Magnetocardiographic functional imaging studies in biomag laboratory. In: et al., C. A. (Ed.), Biomag96, Proc. Tenth Internat. Conf. on Biomagnetism. pp. 494-497.

Nekolla, S., Ibrahim, T., Balbach, T., Klein, C., 2000. Understanding cardiac imaging techniques - from basic pathology to image fusion. IOS Press, Ch. Coregistration and fusion of cardiac magnetic resonance and positron emission tomography studies, pp. 144-154.

Nekolla, S., Miethaner, C., Nguyen, N., Ziegler, S., Schwaiger, M., 1998. Reproducibility of polar map generation and assessment of defect severity and extent assessment in myocardial perfusion imaging using positron emission tomography. Eur. J. Nucl. Med. (25), 1313-1321.
Nenonen, J., Pesola, K., Hänninen, H., K. Lauerma, K., Takala, P., Mäkelä, T. J., Mäkijärvi, M., Knuuti, J., Toivonen, L., Katila, T., 2001. Current-density estimation of exercise-induced ischemia in patients with multivessel coronary artery disease. Journal of Electrocardiography 34 (suppl.), 37-42.

Pallotta, S., Gilardi, M. C., Bettinardi, V., Rizzo, G., Landoni, C., Striano, G., Masi, R., Fazio, F., 1995. Application of a surface matching image registration technique to the correlation of cardiac studies in positron emission tomography by transmission images. Physics in Medicine and Biology 40, 1695-1708.

Papademetris, X., Sinusas, A. J., Dione, D. P., Duncan, J. S., 2001. Estimation of 3D left ventricular deformation from echocardiography. Medical Image Analysis 5, 17-28.

Pesola, K., Lötjönen, J., Nenonen, J., Magnin, I., Lauerma, K., Fenici, R., Katila, T., 2000. The effect of geometry and topology differences in boundary element models on magnetocardiographic localization accuracy. IEEE Trans. Biomedical Eng. 47 (9), 1237-1247.

Pesola, K., Nenonen, J., Fenici, R., Lötjönen, J., Mäkijärvi, M., Fenici, P., Korhonen, P., Lauerma, K., Valkonen, M., Toivonen, L., Katila, T., 1999. Bio-electromagnetic localization of a pacing catheter in the heart. Phys. Med. Biol 44, 2565-2578.

Pham, Q. C., Vincent, F., Clarysse, P., Croisille, P., Magnin, I. E., 2001. A FEM-based deformable model for the 3-D segmentation and tracking of the heart in cardiac MRI. In: Image and Signal Processing and Analysis ISPA 2001. pp. 250-254.

Pluim, J., Maintz, J., Viergever, M., 2000. Image registration by maximization of combined mutual information and gradient information. IEEE Trans. Med. Imaging 19 (8), 809-814.

Press, W., Flannery, B., Teukolsky, S., Vetterling, W., 1988. Numerical recipes in C. Cambridge University Press, Cambridge. 
Sermesant, M., Coudière, Y., Delingette, H., Ayache, N., Désidéri, J., 2001. An electromechanical model of the heart for cardiac image analysis. In: Medical Image Computing and Computer-Assisted Intervention (MICCAI'01). Vol. 2208 of Lecture Notes in Computer Science (LNCS). Springer, pp. 224-231.

Siltanen, P., 1988. Comprehensive Electrocardiology. Oxford: Pergamon Press, Ch. Magnetocardiography.

Sinha, S., Sinha, U., Czernin, J., Porenta, G., Schelbert, H., 1995. Noninvasive assessment of myocardial perfusion and metabolism: feasibility of registering gated $\mathrm{MR}$ and PET images. Am. J. Roentgenol. 36, 301-307.

Slomka, P., Gilbert, A., Stephenson, J., Cradduc, T., 1995. Automated alignment and sizing of myocardial stress and rest scans to threedimensional normal templates using an image registration algorithm. J. Nucl. Med. 36, 11151122 .

Tai, Y.-C., Lin, K., Hoh, C., Huang, S., Hoffman, E., 1997. Utilization of 3-D elastic transformation in the registration of chest X-ray CT and whole body PET. IEEE Trans. Nucl. Med 44 (4), 1606-1612.

Thirion, J.-P., 1995. Fast non-rigid matching of 3-D medical images. Research Report 2547, INRIA.

Thirion, J.-P., 2001. Understanding cardiac imaging techniques - from basic pathology to image fusion. IOS Press, Ch. Perfusion and motion from gated SPECT, pp. 84-93.

Waiter, G. D., Al-Mohammad, A., Norton, M. Y., Redpath, T. W., Welch, A., Walton, S., 2000. Regional myocardial wall thickening assessed at rest by ECG gated (18)F- FDG positron emission tomography and by magnetic resonance imaging. Heart 84, 332-333.

Xu, C., Prince, J. L., March 1998. Snakes, shapes, and gradient vector flow. IEEE Trans. Image Proc. 7 (3), 359-369.
Yu, J. N., Fahey, F. H., Gage, H. D., Eades, C. G., Harkness, B. A., Pelizzari, C. A., December 1995. Intermodality, retrospective image registration in the thorax. J. Nucl. Med. 36 (12), 2333-2338.

Zienkiewicz, O., Taylor, R., 1987. The Finite Element Method. McGraw Hill Book Co. 